\title{
Divergent perspectives on the Dodo bird? A comment to Mullen, Shuluk and Soydan
}

\section{ANDERS BERGMARK \& TOMMY LUNDSTRÖM}

The response from Mullen, Shuluk and Soydan (MSS) is somewhat difficult to answer given the fact that it seems to be based on a misunderstanding of our critique. MSS write that we criticize them "for denying the importance of the "Dodo-Bird" phenomena in explaining social work outcomes". This is not correct, our main critique was directed towards the following standpoint in the report from the National Board of Health and Welfare (NBHW): "Nowadays the aggregate, advanced research on differential treatment effects, shows that the Dodo bird hypothesis in principle has no scientific support" (our translation p. 11). Given the fact that this statement concerns psychotherapy (all the references that are provided by MSS in support of this conclusion, number 10-16, concerns psychotherapy), we described it as highly controversial since it can be said to leave out a substantial amount of research that speaks in favour of the Dodo bird verdict.

In their response MSS points to the fact that the report is based upon a previously published article by Mullen and Shuluk (2011) and "with expanded attention to role of common factors in explaining outcomes written by Soydan ("Dodo-Bird" phenomena")." In the article by Mullen and Shuluk (MS) the conclusions concerning the Dodo bird verdict are quite different in comparison to the report from NBHW. Whereas the latter claims that there is no scientific support for the Dodo bird hypothesis the former (MS) notice "While the dodo's verdict continues to reign, Reid's review (from 1997, our brackets) serves to raise some doubt about its validity when applied to social work."

On a general level we do not have any major objections to MS article, but concerning the understanding of the more recent article by Reid and colleagues, we have a more critical analysis than the one provide by MS. An examination of the material forming the basis for the analysis put forward by Reid and colleagues (2004) on the differential effects of social work interventions, reveals some significant shortcomings. Of the 39 studies selected for analysis only a minority also includes a follow-up after the intervention has been terminated, 27 of the studies compared the effects of the various interventions only at termination (that 
is, without any follow up over time). The absence of a follow-up is a quality shortcoming, which normally leads to an exclusion of the study in a systematic review. If these 27 analyses are removed 12 studies with a reasonable follow-up times remains. While 7 of these show a difference between the interventions, the remaining 5 show no differences. However, the group with the identified differences comprised a sig- nificantly higher proportion of studies of lower quality (quasi-experimental design, 5 of 7) compared to the group that did not identify any differences ( 1 of 5 had a quasiexperimental design). In our view this indicates that both Reid and colleagues (2004) as well as the authors of the national report has drawn conclusions on a material which must be regarded as rather weak.

\section{References}

Mullen, E., Shuluk, J. (2011) Outcomes of social work intervention in the context of evidencebased practice. Journal of Social Work. 11, 49-63.
Reid, WJ, Kenaley, BD \& Colvin, J. (2004) Do some interventions work better than others? A review of comparative social work experiments, Social Work Research, 28, 71-81. 